\title{
Evaluating the use of ERA Mini Dental Implants Retaining Mandibular Overdenture (In Vitro Study)
}

\section{Sabet ME ${ }^{1 *}$, Shawky AMOA ${ }^{2}$ and AlyRagab DAM ${ }^{3}$}

${ }^{1}$ Professor and Chairman of Prosthodontics, Faculty of Dentistry, Ain Shams University, Egypt

${ }^{2}$ Lecturer of Prosthodontics, Faculty of Dentistry, Ain Shams University, Egypt

${ }^{3}$ Clinical instructor at Prosthodontics Department, Faculty of Dentistry, Ain Shams University, Egypt

\begin{abstract}
This simulation study was conducted to evaluate the use of ERA white attachment versus Ball attachment for mini dental implants retained mandibular overdenture regarding retentive forces and forces transmitted to the peri-implant bone during overdenture loading.

This in vitro study was carried out on duplicated educational mandibular acrylic model, and experimental overdentures. In the intraforaminal region, four mini dental implants were installed in each cast. One cast received mini implants with ERA white attachment, while the other received mini implants with ball attachment. The female attachment components were picked up in the overdenture. Strain gauges were installed, one on the distal surface of each of the two distal implants.
\end{abstract}

Universal testing machine was used to apply standardized static load unilaterally on the lower right first molar, both vertically, and obliquely. Micro strains were recorded for each strain gauge. Data obtained were calculated, tabulated, and statistically analyzed to compare between the two attachment designs.

Hooks were then attached to the over dentures, and dislodgment test was performed by the Universal testing machine. Data obtained were calculated, tabulated, and statistically analyzed to compare between the two attachment designs.

The results of this study revealed that the ERA white attachment transmits less strain to the peri-implant bone than the ball attachment, on both vertical and oblique loading while the ball attachment is more retentive then the ERA white attachment.

Keywords: Mandibular overdenture; Dental implants; Prosthodontics

\section{Introduction}

It is assumed that $10 \%$ of the world's population of 6 billion is partially or totally edentulous. This means that there are millions of edentulous people worldwide who need treatment for a condition that can represent considerable disability [1].

Removable denture function in fully edentulous patients is often inadequate. In particular, severe resorption of the alveolar ridges frequently makes it very difficult for patients to wear conventional dentures due to the lack of retention and the instability of the denture. Together with the poor load-bearing capacity of the tissues, this situation can lead to oral pain and discomfort and poor oral function [2].

The use of dental implant to provide support and/or retention for prosthesis offers multiple advantages compared with the use of removable soft tissue restoration [3]. In the last few years, root form implants ranging from $1.8 \mathrm{~mm}$ to slightly more than $2 \mathrm{~mm}$ in diameter have been approved for long-term service [4]. Histologically, the bone appeared to be integrated to the surface of the mini dental implants at the light microscopic level, and the bone appeared to be relatively mature and healthy. The advantages and scientific findings of the smalldiameter commercially pure titanium threaded implant have provided the clinician with a predictable and financially feasible solution for loose dentures [5].

As the mini dental implants are implanted through flapless implant surgery, they possess the advantages of reduced bleeding, decreased postoperative discomfort, and shortened healing time [6]. Also mini implants are immediately loaded, so they allow immediate use of patient's denture after implant placement, thus overcoming the problems of diet difficulties and unaesthetic appearance during healing period between implant placement and final prosthesis [7].
Survival rates reported for mini dental implants range from $83.9 \%$ to $97.5 \%$, depending on location, forces transmitted and whether the implant is used for single-tooth or multi-tooth supported prosthesis [8]. The load transfer mechanism of the implant system is altered significantly by the types of the overdenture attachment [9]. Clinically, those attachment systems that provide the most equitable transfer of occlusal forces among abutments are preferred from the standpoint of bone preservation and implant success [10].

Implant-retained overdentures using ball-type retentive attachments have been shown to be a successful and cost-effective oral rehabilitation approach for edentulous individuals with resorbed mandibular ridges [11]. Sterngold introduced a mini implant using a smaller version of their ERA attachment, and assuming that it transfers less force to the abutment due to its design. Sterngold's ERA Micro overdenture abutment is smaller than the ball attachment. With a built in resiliency that reduces force transmitted to the implant [12].

\section{Materials and Methods}

An impression of the modified educational mandibular edentulous

*Corresponding author: Marwa Ezzat Sabet, Professor and Chairman of Prosthodontics, Faculty of Dentistry, Ain Shams University, Egypt, Tel: 002/01111159878; E-mail: marwa.sabet@yahoo.com

Received December 17, 2013; Accepted Janaury 17, 2014; Published January 19,2014

Citation: Sabet ME, Shawky AMOA, AlyRagab DAM (2014) Evaluating the use of ERA Mini Dental Implants Retaining Mandibular Overdenture (In Vitro Study) Dentistry 4: 196. doi:10.4172/2161-1122.1000197

Copyright: $\odot 2014$ Sabet ME, et al. This is an open-access article distributed unde the terms of the Creative Commons Attribution License, which permits unrestricted use, distribution, and reproduction in any medium, provided the original author and source are credited. 
stone model was made using silicon rubber base impression material (SpeedexColtene/ Whaledent AG, Altstatten/Switzerland). Two identical edentulous mandibular test models were fabricated by pouring the impression twice by molten base plate wax using a mechanical vibrator, and were left to harden. After complete hardening, the casts in wax were removed and processed into pink heat polymerizing acrylic resin.

A sheet of modeling wax was adapted to the ridge and acrlyic resin teeth was set and waxed up. The waxed up overdenture was then transferred to the educational stone model, flasked and processed into heat polymerizing acrylic resin. Finally the experimental overdenture was duplicated to have two identical overdentures.

In each mandibular test model, four mini implants $2.2 \mathrm{~mm}$ in diameter and $13 \mathrm{~mm}$ in length were placed equidistant from one another, to ensure the parallism of the implants a dental surveyor with a hand piece (NOUVAG AG CH-9403 Goldach) was used to drill the implant sites then the implants were fixed with autopolymerizing acrylic resin.

Mini implants for group I were ERA (ERA mini dental implants, Zimmer Dental Inc., Carlsbad, CA, USA)while for group II ball type (Ball mini dental implants 3M ESPE, St. Paul, USA) were used, the attachment systems for each type were picked up into the mucosal surfaces of the experimental overdentures using an autopolymerising acrylic resin.

Attachments were covered with modeling wax to block all undercuts. The cast was boxed in boxing wax, and dental stone was poured over the boxed cast. After setting, the index was separated from the cast. A layer of $2 \mathrm{~mm}$ uniform thickness was removed from the surface of the denture bearing area. This was performed using a $2 \mathrm{~mm}$ diameter round bur to prepare depth cuts in the denture bearing area, then uniform reduction was performed using acrylic bur.

A fissure bur was used to prepare the model to receive the strain gauge $1 \mathrm{~mm}$ distal to each implant. At these sites the acrylic alveolar ridge distal to each implant was reduced and thinned parallel to the implant's long axis, to form a box like chamber of acrylic resin $1 \mathrm{~mm}$ in thickness so that the strain gauges are embedded in these chambers.

In each model four strain gauges (KFG-1-120-C1-11L1M2R, with gauge factor $2.08 \pm 1.0 \%$, guage length $1 \mathrm{~mm}$, guage resistance 120.4 $\pm 0.4 \Omega$, adaptor thermal expansion $11.7 \mathrm{PPM} /{ }^{\circ} \mathrm{C}$, and temperature coefficient of gauge factor $+0.008^{\circ} \mathrm{C}$ ) were installed each $1 \mathrm{~mm}$ distal to each implant. The wires of the strain gauges were oriented vertically in the created chambers and fixed in position using an adhesive recommended by the manufacturer (Figure 1). A medium bodied addition silicon rubber base impression material (AquasilMonophase, Dentsply Caulk, Germany) with nearly the same viscoelasticity of the oral mucosa was injected over the reduced residual ridge. The stone index was painted by separating medium and repositioned to its previous position on the acrylic model till complete setting of the impression material (Figure 2).

\section{Loading tests}

Universal testing machine (LLOYD instruments, Universal Testing Machine) was used to apply unilateral standardized static vertical and oblique load with a magnitude of $100 \mathrm{~N}$ at the right first molar region on the occlusal surface of the experimental overdentures. Oblique loading was performed using a surveyor table (Figure 3).

Micro strains were recorded at each site of the strain gauges. A strain meter was used to assess the strains induced by each applied load.

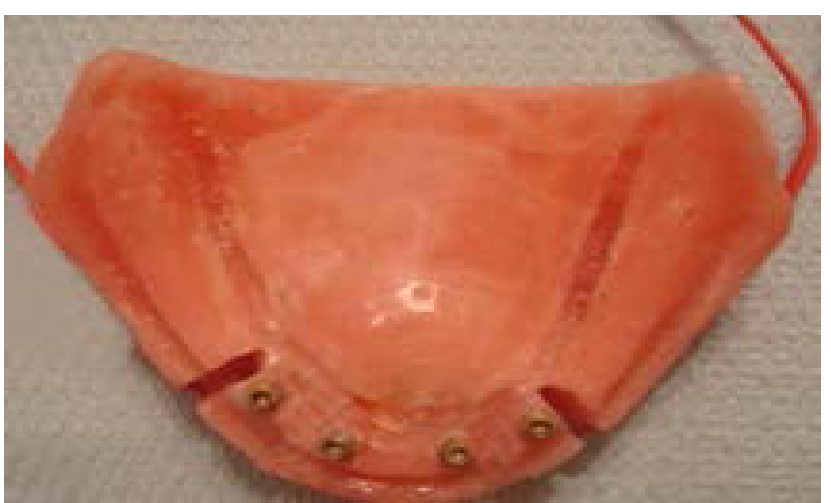

Figure 1: Model 2 with ERA mini implant having the strain gauge wires attached to the model.

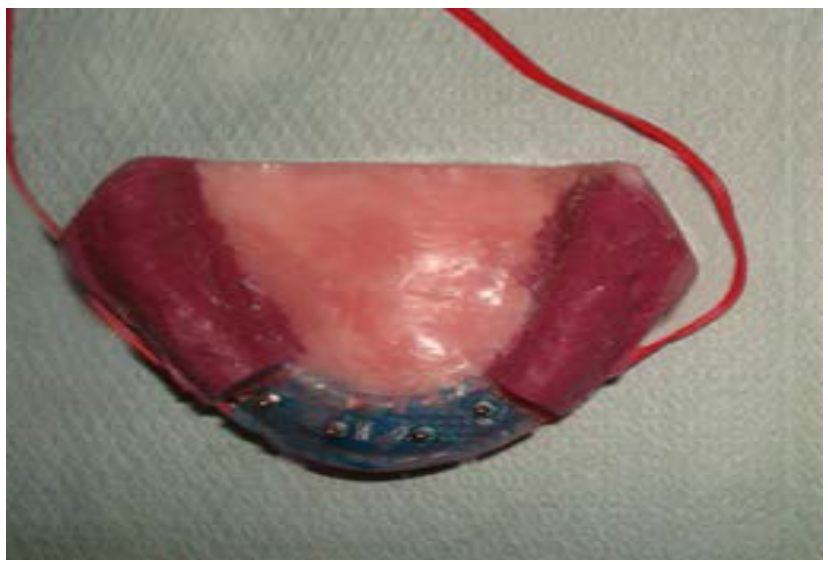

Figure 2: Model 1 having ball type mini implant with simulation of oral mucosa

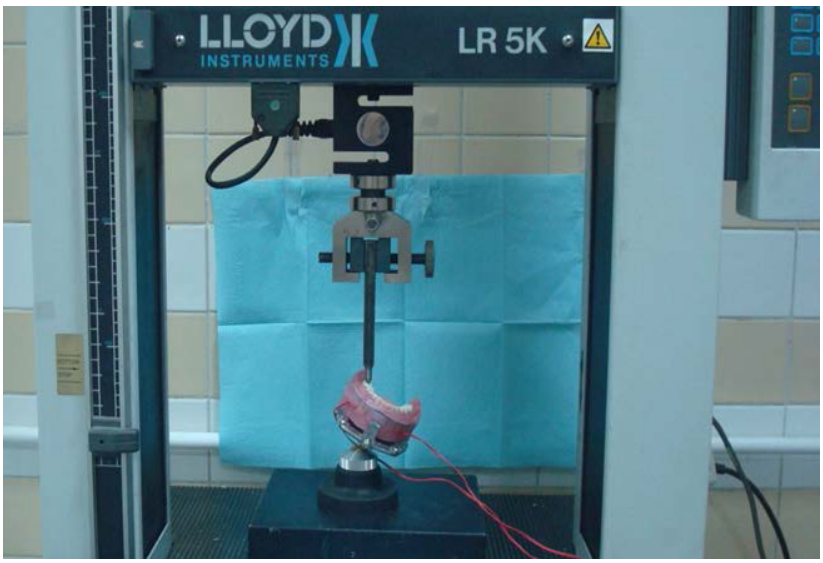

Figure 3: Universal testing machine applying oblique unilateral forces.

The values of the recordings from the strain gauges (strain) were used as an indicator of the stress generated in the peri-implant bone.

\section{Dislodging tests}

Three metal hooks were attached to the occlusal surface of each experimental overdenture in the mid-anterior and bilateral molar regions for chains used in dislodging tests. Overdenture dislodgements were performed using a Universal testing machine (Figure 4). The 


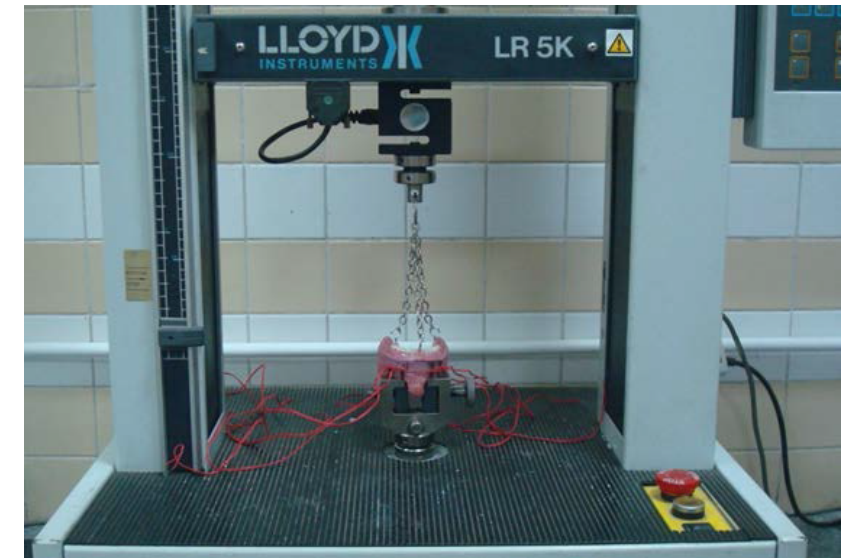

Figure 4: Universal testing machine recording the retentive forces.

crosshead speed was $50 \mathrm{~mm} / \mathrm{min}$. Metallic chains connected the universal testing machine to the overdenture at the withdrawal hooks. Vertical dislodgement was performed with the three metallic chains attached to the hooks in the mid-anterior and bilateral molar regions.

The maximum load needed to dislodge the experimental overdenture from the mandibular test model (retentive force) was recorded. Five measurements were performed for each of the two types of attachment systems.

\section{Results}

The mean values of the recorded micro strains induced under unilateral loading during vertical loading, using ball versus ERA white attachments are shown in Table 1 and Figure 5. Independent t-test was used to compare between the two studied groups.

The results obtained from Table 2 revealed that during vertical loading, the amount of micro strains induced by ERA white attachment is lower than that induced by the ball attachment, that is statistically significant also during oblique loading, the ERA white attachment showed lower micro strains than the ball attachment that were statistically significant $(\mathrm{P} \leq 0.05)$.

The mean values of the retentive forces recorded during vertical dislodgment, using ball versus ERA white attachments are shown in Table 2 and Figure 6. Independent t-test was used to compare between the two studied groups.

\section{Discussion}

During functioning with an implant-retained overdenture, loads are transmitted to alveolar bone surrounding the implants, as well as to the abutments and residual ridges. It is important not to cause unfavorable loads on the implant abutments that house the attachments, as these loads can be detrimental to the osseointegrated implants [13].

The immediate-load nature of the mini implants gives patients an immediate satisfaction without delays in treatment to accommodate conventional healing, permitting full osseointegration. Because mini implants are immediately loaded, it is important to avoid lateral loads on the fixtures that may lead to failure of the implant to integrate and loss of the fixture [14].

The results obtained from this study showed that in both vertical and oblique loading situations, the use of ERA white attachment reduced the strain in the peri-implant bone. This may be attributed to the fact that the height of the head of the ball may allow lateral loads to be placed on the head during insertion or removal of the prosthesis. Sterngold's ERA Micro overdenture abutment is smaller than the ball attachment. With a built in resiliency that reduces force transmitted to the implant. Thus the dampening effect of the ERA attachment is kinder to the implant and surrounding structures. With this in vitro which is also true in vivo for this reason. The ERA implant has a built in spacer which increases the dampening effect of the ERA implant. This is in agreement with Kurtzman and Dompkowski [12].

Since less of the fixture is supercrestal, less lateral load can be placed on the fixtures during function or insertion/removal of the prosthesis. An added benefit is with a lower attachment head, less acrylic needs to be removed from the denture to accommodate the attachment's male than when a ball attachment is used. This is in agreement with Kurtzman and Dompkowski [12]. The lower strain induced on the peri-implant bone by ERA attachment than ball attachment may also be attributed to the difference in the matrix patrix relationship of the two attachments

\begin{tabular}{|l|l|c|c|c|c|c|}
\hline & & \multicolumn{2}{|c|}{ Ball and socket } & \multicolumn{2}{c|}{ ERA white attachment } & P value \\
\hline & & Mean & Sd. & Mean & Sd. & \\
\hline Vertical & loaded & 38.8889 & 5.46453 & 11.1111 & 3.33333 & $0.000^{*}$ \\
\hline Oblique & loaded & 172.2222 & 6.18017 & 129.4444 & 17.03754 & $0.000^{*}$ \\
\hline
\end{tabular}

Table 1: Mean, standard deviation (SD) values and results of Independent $t$ test for comparison between stresses induced on loaded sides using ball versus ERA white attachment during both vertical and oblique loading.

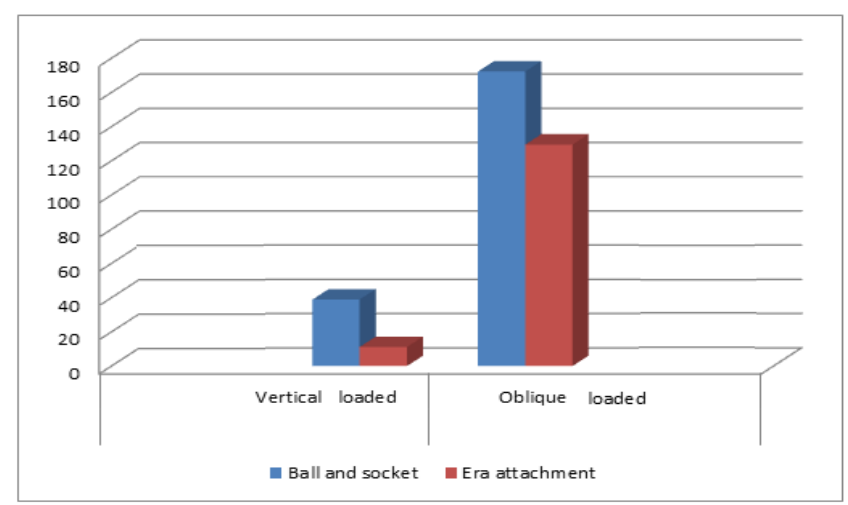

Figure 5: Bar chart representing the mean stress induced on vertical and oblique loading with ball versus ERA white attachment.

\begin{tabular}{|l|c|c|c|c|}
\hline & model & Mean & Std. Deviation & P value \\
\hline retention & ball attachment & 17.9911 & .39808 & $0.000^{*}$ \\
\hline & ERA attachment & 8.5575 & .20313 & \\
\hline
\end{tabular}

Table 2: Mean, standard deviation (SD) values and results of Independent $t$ test for comparison between the retentive forces recorded using ball versus ERA white attachment during vertical dislodgment .

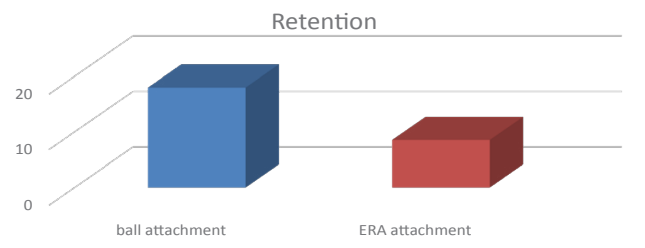

Figure 6: Bar chart representing the mean stress induced on vertical and oblique loading with ball versus ERA white attachment. 
Citation: Sabet ME, Shawky AMOA, AlyRagab DAM (2014) Evaluating the use of ERA Mini Dental Implants Retaining Mandibular Overdenture (In Vitro Study). Dentistry 4: 197. doi:10.4172/2161-1122.1000197

around which the denture was rotating. The ERA attachment design transferred the fulcrum point close to the fixture, thus reducing lever arm and torque and allowing less strain on peri-implant bone which is in accordance with Jingade et al. [15].

The results obtained from this study also showed that the ball attachment is more retentive then the ERA white attachment. This was found in accordance with Petropoulos and Mante which may be attributed to the greater surface area of the ball attachment, as well as its circumferential undercut. The increased resiliency of the ERA white attachment also decreased its retention [16]. In addition, it has to be mentioned that in this in vitro study four $100 \%$ parallel ball implants and four $100 \%$ parallel ERA implants were placed in a mandibular acrylic model which is not the case in vivo. In placing four ball implants or four ERA implants in vivo it is difficult to achieve $100 \%$ parallelism. The margin of error for the ball implant is a maximum of $14 \%$. The manufacturers of the ERA implant give a margin of error of $7 \%$ off parallelism. It can be assumed the ball implant would be more retentive also because greater margin of error for parallelism and the ease of complete seating, whereas the ERA implant if off parallelism would not completely seat and thereby reduce retention. By the nature of the ERA implant it would take only one of the four implants to be off parallelism to prevent complete seating of the denture thereby reducing retention.

\section{Conclusion}

From the results of this study, it could be concluded that the ERA white attachment transmits less stresses to the peri-implant bone on both vertical and oblique loading but Ball attachment is more retentive than ERA white attachment

\section{References}

1. Carlsson GE, Omar R (2010) The future of complete dentures in oral rehabilitation. A critical review. J Oral Rehabil 37: 143-156.

2. Suzuki Y, Osada H, Kobayashi M, Katoh M, Kokubo Y, et al. (2012) Long-term clinical evaluation of implant over denture. J Prosthodont Res 56: 32-36.
3. Doundoulaks JH, Eckert SE, Lindquist CC, Jeffcoat MK (2003) The implantsupported overdenture as an alternative to the complete mandibular denture. $J$ Am Dent Assoc 134: 1455-1458.

4. Christensen GJ (2006) The mini implant has arrived. J Am Dent Assoc 137 387-390.

5. Balkin BE, Steflik DE, Naval F (2001) Mini dental implant insertion with autoadvance technique for ongoing applications. J Oral Implantol 27: 32-37.

6. Campelo L, Camara J (2002) Flapless implant surgery, a 10 year clinical retrospective analysis. Int J Oral Maxillofac Implants 17: 271-276.

7. Ahn MR, An KM, Choi JH, Sohn DS (2004) Immediate loading with mini dental implants in the fully edentulous mandible. Implant Dent 13: 367-372.

8. Griffitts TM, Collins CP, Collins PC (2005) Mini dental implants: an adjunct for retention, stability, and comfort for the edentulous patient. J Oral Surg Oral Med Oral Pathol Oral Radiol Endod 100: 81-84.

9. Chun HJ, Park DN, Han CH, Heo SJ, Heo MS, et al. (2005) Stress distributions in maxillary bone surrounding overdenture implants with different overdenture attachments. J Oral Rehabil 32: 193-205.

10. Porter JA, Petropoulos VC, Brunski JB (2002) Comparison of Load Distribution for Implant Overdenture Attachments. Int J Oral Maxillofac Implants 17: 651662.

11. Naert I, Alsaadi G, Quirynen M (2004) Prosthetic aspects and patient satisfaction with two implant-retained overdentures: a 10-year randomized clinical study. Int J Prosthodont 17: 401-410.

12. Kurtzman GM, Dompkowski D (2012) Improving denture stabilization with the ERA Mini implant. Implant practice 3: 32-37.

13. Zarb G, Bolender C (2004) Prosthodontic Treatment for Edentulous Patients Complete Dentures and Implant-Supported Prostheses. (12thedn), St Louis Mosby.

14. Nazarian A (2005) Mini dental implants: immediate gratification for patient and provider. Dent Today 24: 110-112.

15. Jingade RK, Rudraprasad IV, Sangur R (2005) Biomechnics of dental implants: A FEM study. J Indian Prosthodont Soc 5: 18-22.

16. Petropoulos VC, Mante FK (2011) Comparison of Retention and Strain Energies of Stud Attachments for Implant Overdentures. J Prosthodont 20: 286-293. 